\title{
Imagery Signal-Based Deep Learning Method for Prescreening Major Depressive Disorder
}

\author{
Hyunjin Kwon (D), Jinhyeok Park (D), Seokhwan Kang (D, \\ and Youngho Lee ${ }^{(\bowtie)}$ (D) \\ Department of Computer Engineering, College of IT, Gachon University, \\ 1342 Seongnam-daero, Seongnam-si, Republic of Korea \\ euleekwon@gmail.com, rev.hyeok@gmail.com, \\ \{shkang, 1yh\}@gachon. ac.kr
}

\begin{abstract}
Depression is a high-risk mental illness that can lead to suicide. However, for a variety of reasons, such as a negative perception of mental illness, most patients with depressive symptoms are reluctant to go to the hospital and miss appropriate treatment. Therefore, a simple prescreening method that an individual can use to identify depression is needed. Most EEG measurement devices that individuals use have few channels. However, most studies using EEG to diagnose depression have been conducted in a professional multichannel EEG environment. Therefore, it is difficult for individuals to prescreen depression based on the results of the studies. In this study, we proposed a model that predicts depression by using EEG data measured by a few channels so that it can measure depression using the EEG data measured by an individual. In this study, brain waves measured in two channels were imaged using STFT transform and a spectrogram. The EEG image data was then used in a deep learning model. As a result of the performance evaluation, $75 \%$ accuracy was shown for the classification of image depression EEGs and normal image type EEGs. As a result, low channel EEG data for deep learning can be used as an auxiliary tool to proactively diagnose depressed patients.
\end{abstract}

Keywords: Major depressive disorder - Short-time Fourier Transform • Convolution Neural Network

\section{Introduction}

Major depressive disorder (MDD), which is usually called depression, is a very serious mental illness that can lead to suicide in severe cases. According to the World Health Organization (WHO), more than 300 million people worldwide suffer from depression, and about 800,000 people commit suicide each year [1]. In this situation, Korea has the highest suicide rate among OECD member countries, and the suicide rate is also continuously increasing [2].

Although early detection and appropriate treatment of a large number of mental illnesses, including depression, are very important, the mental health service utilization 
rate in Korea is only about $22.2 \%$, which is very low compared to the United States $(43.1 \%)$ and Canada (46.5\%) [3]. One of the biggest reasons for Korea's low utilization of mental health services is that the country's social perception of all mental illnesses is very negative [4]. Thus, the actual rate of patients experiencing depression is expected to be higher than reported.

Previously, the Hamilton Depression Rating Scale and the Beck Depression Inventory were used to diagnose depression. In recent years, however, several studies have been reported to analyze depression by analyzing brain waves as a result of the development of brain science. Since the field of brain science research became active, inexpensive and simple brainwave measuring devices have emerged to replace expensive conventional brainwave measuring devices. This makes it easy for anyone to measure brainwave data, and the field of brainwave research is no longer limited to specific experts. In other words, this means not only that public access to EEG measurement is improved, but also patients who have depression but refuse to measure EEG in a hospital can measure EEG individually.

An infrastructure where people who have symptoms of depression can simply measure their brain waves at home is gradually being built. However, most of the existing studies that diagnose depression have been conducted with professional brainwave measuring devices that have multiple channels. Therefore, it is difficult for individuals to diagnose depression at home based on the results of these studies. In this study, we proposed a model that can effectively diagnose depression based on the small number of channels of brainwave measuring devices commonly used in the home. In our model, we converted EEG brainwaves to image data and used it for classifying depressed patients and controls. Then we evaluated the performance of classification.

\section{Method}

\subsection{Dataset}

This study was conducted using a set of brainwave data measured in Wajid Mumtaz's study [5]. The data are measured on the brain waves of 34 depressed patients (17 males and 17 females) and 30 healthy controls (21 males and nine females). The brainwaves were measured in the state in which subjects closed their eyes $(5 \mathrm{~min})$, the state in which they opened their eyes $(5 \mathrm{~min})$, and the state in which certain visual contents were progressed $(10 \mathrm{~min}$ ). Brain waves were measured on 19 channels according to the international 10-20 system, where the 19 channels corresponded to the frontal (Fp1, F3, F7, Fz, Fp2, F4, and F8), the central (C3, C4 and Cz), the parietal lobe (P3, Pz and $\mathrm{P} 4)$, the occipital $(\mathrm{O} 1, \mathrm{O} 2)$ and left and right temporal regions (T3, T4, T5 and T6) [6]. In order to use the same number of EEG data, we used 30 brain waves from 30 depressed patients and 30 healthy controls. In addition, we used only Fp1 and Fp2 brainwave data among 19 channels, as low-channel brainwave measuring instruments (Muse and Neuro-Harmony) commonly measure brain waves on two channels (Fp1, Fp2). To minimize noise caused by blinking or external stimuli, we used EEG data measured with eyes closed. 


\subsection{Overview of Research Process}

The flow of this study is as follows (see Fig. 1).

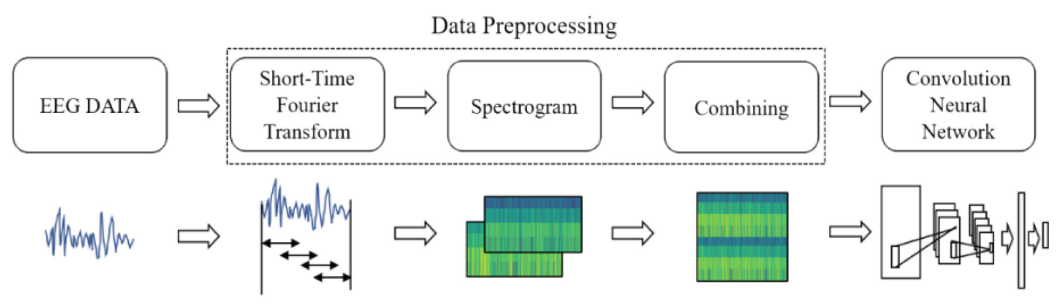

Fig. 1. Research flow

Short-time Fourier transform (STFT) was used as a method to simultaneously express both frequency domain analysis and time domain analysis. STFT is a method of performing Fourier transform on each part by shortening the time to analyze time and frequency simultaneously. The STFT transformation used the optimal parameter values presented in previous studies of the institution that provided this data. The optimal parameters proposed in the previous study are when the window length is $2 \mathrm{~s}$, the hop size is $0.5 \mathrm{~s}$, the FFT point number is $16 \mathrm{~s}$, the sampling frequency is $256 \mathrm{~Hz} / \mathrm{s}$, and the overlap is set to 50\% [5]. In this study, STFT conversion was performed for each channel using the corresponding parameters. We then used a spectrogram to image the results. The following figure shows the results of electroencephalogram (EEG) data obtained from a depressed patient measured on real channel 1 after performing STFT conversion and spectrogram (see Fig. 2).

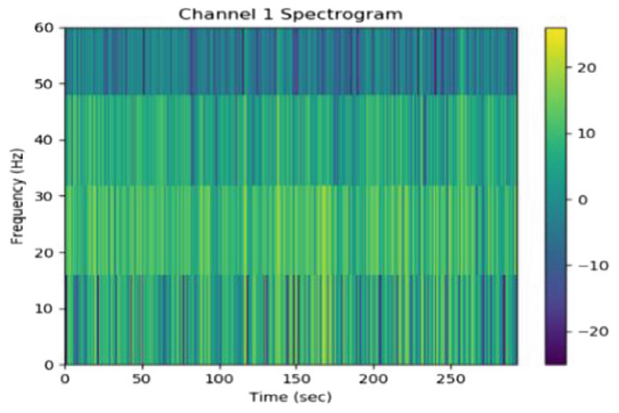

Fig. 2. Signal image of MDD patient

All patients' EEG data were imaged through the spectrogram and then combined into one for use as a single image. Therefore, the electroencephalogram image measured by one channel 1 and the electroencephalogram image measured by channel 2 were expressed as one image. Combined images were categorized as learning data and test data for use in deep learning. The data used for the study were 20 depressed EEG 
image data and 20 healthy EEG image data. The data used for the test were 14 pieces of data using seven pieces of EEG image data. Data were analyzed using the Convolution Neural Network (CNN) model as a deep learning model (see Table 1). Among the ways to classify brain waves, $\mathrm{CNN}$ is known to be very powerful in feature learning ability [7]. The CNN used in this study consisted of a three-layer convolution layer and used the ReLU activation function. We used max pooling with $3 \times 3$ spacing of 2 and soft-max as an activation function in the output stage.

Table 1. Convolution Neural Network model

\begin{tabular}{l|l|l}
\hline Layer (type) & Out shape & Param \# \\
\hline Conv2d_1 (Conv2D) & (None, 62,94,32) & 896 \\
\hline Max_pooling2d_1 (MaxPooling2D) & (None, 31,47,32) & 0 \\
\hline Conv2d_2 (Conv2D) & (None, 29,45,64) & 18496 \\
\hline Max_pooling2d_2 (MaxPooling2D) & (None, 14,22,64) & 0 \\
\hline Conv2d_3 (Conv2D) & (None, 12,20,128) & 73856 \\
\hline Max_pooling2d_3 (MaxPooling2D) & (None, 6,10,128) & 0 \\
\hline Flatten_1 (Flatten) & (None, 7680) & 0 \\
\hline dense_1 (Dense) & (None, 512) & 3932672 \\
\hline dense_2 (Dense) & (None, 2) & 1026 \\
\hline Total params: 4,026,946 & & \\
\hline Trainable params: 4,026,946 & & \\
\hline Non-trainable params: 0 & & \\
\hline
\end{tabular}

The image used for CNN was $64 \times 96$, which is one-hundredth the size of the original image. The batch size was set to 4, the steps per Epoch were set to 10, and Epoch was set to 35 . The number of verification steps used was set to 2 .

\section{Results}

We found that the model had a predictive accuracy of $75 \%$ for classifying image type EEG and healthy image type EEG in patients with depression. The following graphs show the change of performance according to the progress of Epoch. As can be seen in the graph of Fig. 3, the accuracy of the verification increases as the training accuracy

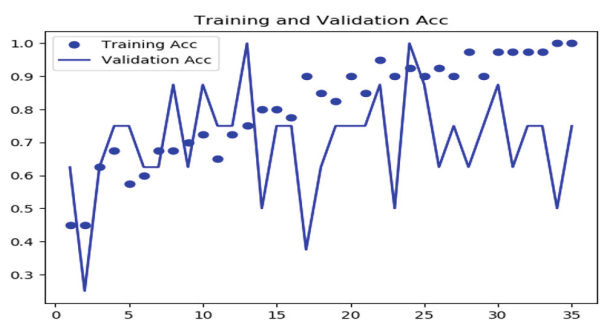

Fig. 3. Accuracy per Epoch 
increases. In addition, in the graph of Fig. 4, the verification loss value decreases as the training loss value decreases.

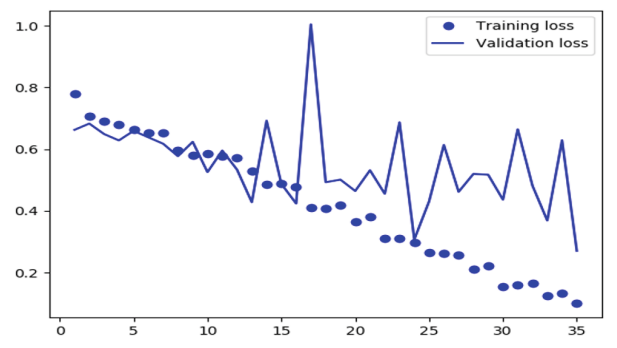

Fig. 4. Loss per Epoch

\section{Discussion and Conclusion}

It is very important to detect, treat and manage depression early. However, most patients with depression refuse to visit the hospital and are often reluctant to undergo a depression diagnostic test. Therefore, effective depression prescreening aids are needed to analyze depression using simple brainwave measuring device results without having to visit the hospital. In this study, we proposed a model that uses image-type brain waves to effectively distinguish patients with depression with data from a small number of channels. Brainwave data from two channels were imaged through a spectrogram after using STFT techniques. The imaging brainwave data were analyzed using the CNN model, which eventually showed a predictive accuracy of $75 \%$.

In this study, we have shown that the method of classifying depressive patients using deep learning based on image EEG data can be used as a prescreening tool for depression. However, considering that the amount of data is one of the most important factors for the performance of deep learning, it is considered that the small number of EEG data used in this study has limitations in deriving the optimum performance of the model. Therefore, in future research, after acquiring a larger number of brain wave data, we will try to confirm its performance by using various deep learning models.

Acknowledgement. This research was supported by the Ministry of Science and ICT (MSIT), Korea, under the Information Technology Research Center (ITRC) support program (IITP-20192017-0-01630) supervised by the Institute for Information \& communications Technology Promotion (IITP).

\section{References}

1. WHO. https://www.who.int/news-room/fact-sheets/detail/depression. Accessed 18 Mar 2019

2. Kwon, J., Chun, H., Cho, S.: A closer look at the increase in suicide rates in South Korea from 1986-2005. BMC Public Health 9(1), 72 (2009) 
3. Ministry of health and welfare. http://www.mohw.go.kr/react/jb/sjb030301vw.jsp?PAR_ MENU_ID=03\&MENU_ID=032901\&CONT_SEQ=339138\&page=1. Accessed 21 Mar 2019

4. Shin, K.: A study on the legal issues and challenges of insurance discrimination against the mentally III. J. Korean Neuropsychiatr. Assoc. 53(2), 61-71 (2014)

5. Mumtaz, W., Xia, L., Yasin, M., Ali, S., Malik, A.: A wavelet-based technique to predict treatment outcome for major depressive disorder. PLoS One 12(2), e0171409 (2017)

6. Klem, G., Lüders, H., Jasper, H., Elger, C.: The ten-twenty electrode system of the international federation. Electroencephalogr. Clin. Neurophysiol. 52(3), 3-6 (1999)

7. Wen, T., Zhang, Z.: Deep convolution neural network and autoencoders-based unsupervised feature learning of EEG signals. IEEE Access 6, 25399-25410 (2018) 